\title{
Amphiphilic core-sheath structured composite fiber for comprehensively performed supercapacitor
}

\author{
Xuemei $\mathrm{Fu}^{1}$, Zhuoer $\mathrm{Li}^{1}$, Limin $\mathrm{Xu}^{1}$, Meng Liao ${ }^{1}$, Hao Sun ${ }^{2}$, Songlin Xie ${ }^{1}$, Xuemei Sun ${ }^{1}$, \\ Bingjie Wang ${ }^{1^{*}}$ and Huisheng Peng ${ }^{1 *}$
}

\begin{abstract}
As an important branch of fiber-shaped energy storage devices, the fiber-shaped supercapacitor has been widely studied recently. However, it remains challenging to simultaneously achieve fast electron transport and excellent ion accessibility in one single fiber electrode of the fibershaped supercapacitor. Herein, a novel family of amphiphilic core-sheath structured carbon nanotube composite fibers has been developed and applied to the fiber-shaped supercapacitor to address the above challenge. The polyaniline-modified hydrophilic sheath of the composite fiber electrode effectively enhanced the electrochemical property via advancing ion accessibility, while Au-deposited hydrophobic core demonstrated improved electrical conductivity by fast electron supply. On the basis of a synergistic effect, a remarkable specific capacitance of $324 \mathrm{~F} \mathrm{~cm}^{-3}$ at $0.5 \mathrm{~A} \mathrm{~cm}^{-3}$ and greatly enhanced rate performance were achieved, i.e., a $79 \%$ retention $\left(256 \mathrm{~F} \mathrm{~cm}^{-3}\right)$ at $50 \mathrm{~A} \mathrm{~cm}^{-3}$. The obtained fiber-shaped supercapacitor finally displayed remarkable energy and power densities of $7.2 \mathrm{~mW} \mathrm{~h} \mathrm{~cm}$ and $10 \mathrm{~W} \mathrm{~cm}^{-3}$, respectively. The strategy developed herein also presents a general pathway towards novel fiber electrodes for high-performance wearable devices.
\end{abstract}

Keywords: hydrophobicity, hydrophilicity, amphiphilic coresheath, supercapacitor, electron transport, ion accessibility

\section{INTRODUCTION}

The emerging wearable electronics has driven the development of fiber-shaped energy devices in recent years [1-3]. As an important branch, fiber-shaped supercapacitor with diameters of tens to hundreds of micrometers can efficiently accommodate complex deformations, and further be woven into soft, breathable and comfortable textiles to realize fast charging of various commercial electronics [4-6]. Fiber electrodes, as the key part of fiber-shaped supercapacitors, are widely explored based on carbon nanomaterials such as carbon nanotubes (CNTs) and graphene fibers due to their high mechanical strength, electrical conductivity and specific surface area [7-11]. The incorporation of carbon nanomaterials with other pseudocapacitive materials such as transition metal oxides and conducting polymers is a common strategy to pursue higher energy density [12-16]. For instance, a micro-supercapacitor based on microfluidic-oriented core-sheath polyaniline (PANI) nanorod arrays/graphene composite fibers demonstrated high energy density of $37.2 \mathrm{~mW} \mathrm{~h} \mathrm{~cm}^{-2}$ [15]. However, the pseudocapacitance is far from fully developed especially at high rate owing to the insufficient electron supply and ion accessibility during electrochemical reactions. As a result, it is important to develop new fiber electrodes to realize efficient electron supply and ion accessibility simultaneously.

Here, a new family of amphiphilic core-sheath structured CNT fibers has been designed. The core was made from pristine CNTs incorporated with $\mathrm{Au}$ nanoparticles, and the sheath consisted of oxygen plasma-functionalized hydrophilic CNTs (OCNTs). The hydrophilic fiber surface not only benefits the deposition of a second phase but also promotes the redox reactions in common aqueous systems [17-19]. As a demonstration, the core-sheath structured fiber was used as substrate to electrodeposit PANI, denoted as CNTAu@OCNT-PANI (Fig. 1a). Impressively, the CNTAu@OCNT-PANI demonstrated both high specific capacitance and rate capability, i.e., $256 \mathrm{~F} \mathrm{~cm}^{-3}$ at a high current density of $50 \mathrm{~A} \mathrm{~cm}^{-3}$ with a $79 \%$ retention of that at $0.5 \mathrm{~A} \mathrm{~cm}^{-3}\left(324 \mathrm{~F} \mathrm{~cm}^{-3}\right)$. A remarkable energy density

\footnotetext{
${ }^{1}$ Laboratory of Advanced Materials, State Key Laboratory of Molecular Engineering of Polymers and Department of Macromolecular Science, Fudan University, Shanghai 200438, China

2 Department of Chemistry, Stanford University, California 94305, the United States

* Corresponding authors (emails: wangbingjie@fudan.edu.cn (Wang B); penghs@fudan.edu.cn (Peng H)).
} 


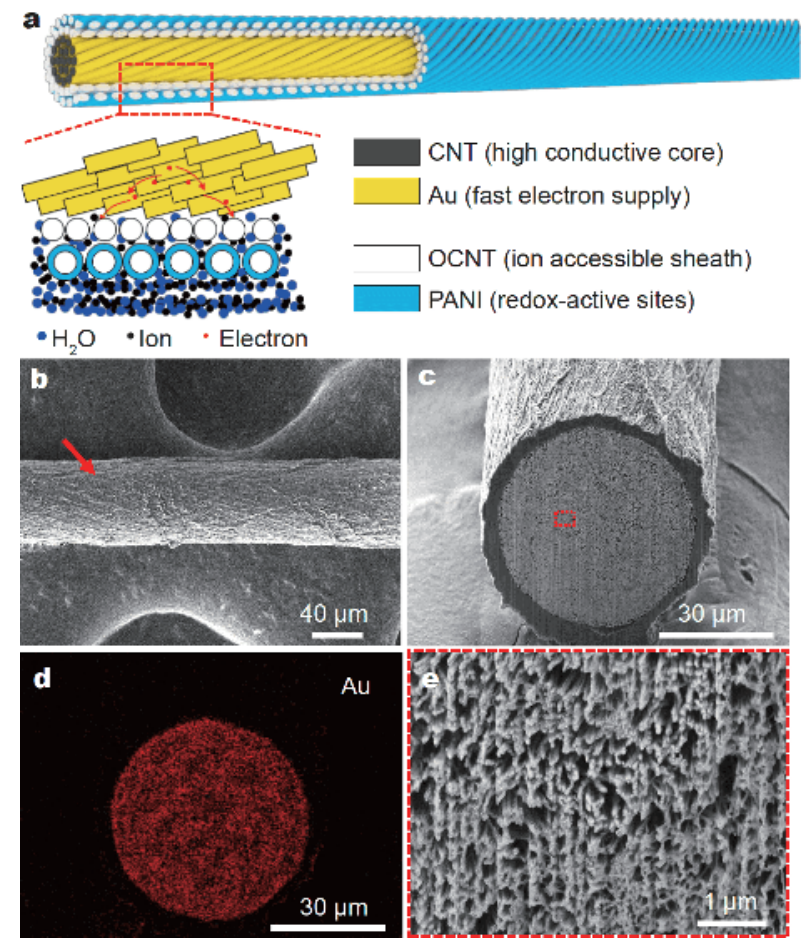

Figure 1 Structure and composition of the amphiphilic core-sheath structured composite fiber. (a) Schematic illustration to the amphiphilic core-sheath structured CNT-Au@OCNT-PANI fiber electrode. (b) PANI modified surface of the composite fiber. (c) Cross-sectional SEM image of the fiber electrode with PANI modified OCNTs outside and Au deposited CNTs inside. (d) EDS element mapping of Au in (c). (e) SEM image showing $\mathrm{Au}$ distribution corresponding to the red rectangle in (c).

and power density of $7.2 \mathrm{~mW} \mathrm{~h} \mathrm{~cm}^{-3}$ and $10 \mathrm{~W} \mathrm{~cm}^{-3}$ were achieved in the resulting fiber-shaped supercapacitors, respectively.

\section{EXPERIMENTAL SECTION}

\section{Preparation}

\section{Preparation of pristine CNTs and OCNTs}

Pristine hydrophobic CNT sheets were drawn from the spinnable CNT array which was obtained by chemical vapor deposition [10]. The CNT sheets were deposited on a two-ends elevated glass slide, and then were subjected to oxygen plasma treatment with different time under a power of $100 \mathrm{~W}$ with a RF frequency of $13.56 \mathrm{MHz}$ (CIF, CPC-C), in order to produce hydrophilic OCNT sheets.

Preparation of core-sheath structured CNT-Au@OCNTPANI composite fibers

$\mathrm{Au}$ nanoparticles were deposited on the pristine CNT sheets by a three rotary target plasma sputtering coater
(MTI Corp., VTC-16-3HD) with deposition time of $300 \mathrm{~s}$ under the pressure of $10 \mathrm{~Pa}$. Then the resulting CNT-Au sheets were twisted into a fiber using a motor equipped with a sharp head. Afterwards, the OCNT sheets were wound around the above-mentioned CNT-Au fiber, followed by modification with PANI through electrodeposition using $\mathrm{Ag} / \mathrm{AgCl}$ electrode and platinum wire as the reference and counter electrodes in aqueous electrolyte, respectively. The electrolyte consisted of $0.1 \mathrm{~mol} \mathrm{~L}^{-1}$ aniline and $1 \mathrm{~mol} \mathrm{~L}^{-1} \mathrm{H}_{2} \mathrm{SO}_{4}$. The loading of PANI was controlled by the consumed charge during the electrodeposition process at constant voltage of $0.75 \mathrm{~V}$, according to the ratio versus the total mass of CNTs and OCNTs. After being washed by deionized water, the composite fibers were dried at room temperature.

\section{Preparation of fiber-shaped supercapacitor}

The polyvinyl alcohol (PVA) $/ \mathrm{H}_{3} \mathrm{PO}_{4}$ gel electrolyte was prepared by adding PVA powder $(1 \mathrm{~g})$ to $9 \mathrm{~mL}$ deionized water, followed by vigorous stir under $90^{\circ} \mathrm{C}$ until the mixture became clear. After being cooled down to room temperature, $\mathrm{H}_{3} \mathrm{PO}_{4}(\mathrm{~g})$ was dropped into the mixture. Two core-sheath structured CNT-Au@OCNT-PANI fibers were coated with the gel electrolyte $(\sim 200 \mu \mathrm{m}$ thick) and intertwined together to fabricate a fiber-shaped supercapacitor, and the fiber-shaped supercapacitor can be inserted into a plastic tube with two ends sealed with UV curable resin to realize steady operation.

\section{Characterization}

The morphology and structure of the composite fibers were characterized by a field-emission scanning electron microscope (SEM) operated at $5 \mathrm{kV}$ (Ultra 55, Zeiss) with energy dispersive X-ray spectroscopy (EDS) element mapping operated at $15 \mathrm{kV}$. Electrical conductivities were tested by a Keithley 2400 Source Meter. Electrochemical measurements and electrodeposition were completed on an electrochemical workstation (CHI 660E) except that electrochemical impedance spectroscopy (EIS) in a threeelectrode system was conducted on Autolab PGSTAT204 equipped with FRA32M EIS module (Metrohm). In the three-electrode system, platinum wire and saturated calomel electrode (SCE) were used as the counter and reference electrodes, respectively, with $1 \mathrm{~mol} \mathrm{~L}^{-1} \mathrm{H}_{3} \mathrm{PO}_{4}$ as electrolyte. The cross sections were obtained by cutting the core-sheath fibers with a focused $\mathrm{Ga}$ ion beam using $30 \mathrm{kV}$ voltage and $20 \mathrm{nA}$ beam current (Zeiss Auriga), followed by polishing using ion beam with currents ranging from 1 to $4 \mathrm{nA}$. Raman spectroscopy was recorded by XploRA (HORIBA JobinYvon). The UV-vis 
spectroscopy was obtained via UV-2550 from Shimadzu. Photographs were captured by a camera (A5000, Sony).

\section{Calculation}

All the calculation of electrochemical parameters was based on two-electrode system. The gravimetric capacitance $\left(C_{\mathrm{M}}, \mathrm{F} \mathrm{g}^{-1}\right)$ was calculated from $C_{\mathrm{M}}=2 \times I \times \Delta t /(\Delta V$ $\times m)$, while the volumetric capacitance $\left(C_{\mathrm{V}}, \mathrm{F} \mathrm{cm}^{-3}\right)$ was from $C_{\mathrm{V}}=2 \times I \times \Delta t /(\Delta V \times V)$. Here $I(\mathrm{~A}), \Delta t(\mathrm{~s})$ and $\Delta V$ (V) refer to the discharge current, discharge time, and voltage window, respectively. And $m(\mathrm{~g})$ and $V\left(\mathrm{~cm}^{3}\right)$ correspond to the mass and volume of the whole electrode, i.e., CNTs, Au, OCNTs and PANI. For the fiber-shaped supercapacitor, the volumetric energy

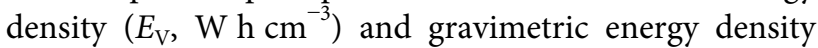
$\left(E_{\mathrm{M}}, \mathrm{Wh} \mathrm{g}^{-1}\right)$ were calculated from $E_{\mathrm{V}}=C_{\mathrm{V}} \times \Delta V^{2} /(8 \times$ $3,600)$ and $E_{\mathrm{M}}=C_{\mathrm{M}} \times \Delta V^{2} /(8 \times 3,600)$, respectively. The volumetric power density $\left(P_{\mathrm{V}}, \mathrm{W} \mathrm{cm}{ }^{-3}\right)$ and gravimetric power density $\left(P_{\mathrm{M}}, \mathrm{W} \mathrm{g}^{-1}\right)$ were calculated according to $P_{\mathrm{V}}=E_{\mathrm{V}} \times 3,600 / \Delta t$ and $P_{\mathrm{M}}=E_{\mathrm{M}} \times 3,600 / \Delta t$, respectively.

\section{RESULTS AND DISCUSSION}

The structure and morphology of the CNT-Au@OCNTPANI fibers are illustrated in Fig. 1. The designed CNTAu@OCNT-PANI fiber has a core-sheath structure with enhanced ion accessible surface across the sheath and fast electron supply across the core (Fig. 1a). PANI was uniformly deposited on the hydrophilic OCNT sheath via an electrochemical method (Fig. 1b and Fig. S1). Here, hydrophilic OCNTs were obtained by oxygen plasma treatment on the pristine CNTs. Cross-sectional SEM image exhibits a typical core-sheath structure in which the brighter core is the Au-deposited CNTs and the darker surround ring is the hydrophilic OCNTs modified by PANI (Fig. 1c). The electrode core preserved the high conductivity and mechanical strength of the pristine CNTs [20], and the introduction of $\mathrm{Au}$ nanoparticles further enhanced the electrical conductivity. EDS element mapping indicates that the $\mathrm{Au}$ nanoparticles were incorporated uniformly in the CNT core (Fig. 1d). In the magnified SEM image of CNT-Au core (Fig. 1e), CNT bundles can still be clearly observed, suggesting that $\mathrm{Au}$ nanoparticles were deposited on the single CNT or CNT bundles.

To prove the superiority of hydrophilic sheath, we investigated the effect of hydrophilic/hydrophobic surface on the deposited morphology of conducting polymer in the aqueous system. Different contents of PANI were electrodeposited on the CNT fibers with hydrophobic (CNT@CNT-PANI) and hydrophilic (CNT@OCNT-
PANI) sheaths. The consuming time for certain charge number equivalent to the PANI loading varied distinctly due to the difference of hydrophobic CNTs and hydrophilic OCNTs (Fig. S2). It was faster for fibers with hydrophilic OCNT to deposit the same content of PANI with a higher initial current (Fig. S3). Then the surface morphology and structure of the CNT@CNT-PANI and CNT@OCNT-PANI with increasing PANI contents from 10 to 70 wt.\% were compared (Fig. $2 \mathrm{a}-\mathrm{d}$ and Fig. S4). At the PANI content of $10 \mathrm{wt} . \%$, the two composite fibers show similar surface morphology with few PANI on the CNT bundles (Fig. S4a, d). As PANI loadings increased over 30 wt.\%, CNT@CNT-PANI fibers show bulky aggregation of PANI, indicating that more PANI tended to deposit on the existing composite sites or in the pore space among CNTs (Fig. S4b, c). For the CNT@OCNTPANI fibers, more oxygen-containing groups and defect sites on the hydrophilic OCNT sheath were beneficial to the infiltration and electrochemical polymerization of aqueous aniline solution, facilitating the binding between OCNTs and PANI. As a result, in the electrodeposition process, more PANI deposited on the OCNTs directly with clear CNT bundles on the surface and negligible aggregation of PANI observed until the loading increased to $50 \mathrm{wt} . \%$ (Fig. S4e, f). The low and high magnified images of CNT@CNT-PANI and CNT@OCNT-PANI with 70 wt.\% content of PANI show a striking contrast in Fig. 2a-d. For CNT@CNT-PANI fibers, excess PANI aggregates on the outer surface of composite fibers (Fig. 2a, b). However, even with 70 wt.\% PANI, CNT bundles and the original oriented structure were still observed clearly on the CNT@OCNT-PANI fibers (Fig. 2c). In the magnified SEM image, the PANI was uniformly deposited on the CNT bundles (Fig. 2d), indicating good affinity between OCNTs and PANI. For the CNT@OCNT-PANI fibers, OCNTs with increased time from 1 to $3 \mathrm{~min}$ of oxygen plasma treatment show better affinity between OCNTs and PANI (Fig. S5), for the increasing treatment time leads to more hydrophilic OCNTs. However, the increasing time of oxygen plasma treatment generates more defects which can harm the electrical conductivity and mechanical strength of the resulting fibers [20].

Raman spectra revealed stronger interactions between OCNTs and PANI than those between CNTs and PANI (Fig. 2e). The Raman spectra of pure OCNTs and CNTs were also plotted to show the typical peaks at about 1,320 and $1,560 \mathrm{~cm}^{-1}$ assigned to $\mathrm{D}$ band indicative of defects and $\mathrm{G}$ band indicative of graphitic carbon, respectively. Obvious increase of D band peak intensity for OCNT at 

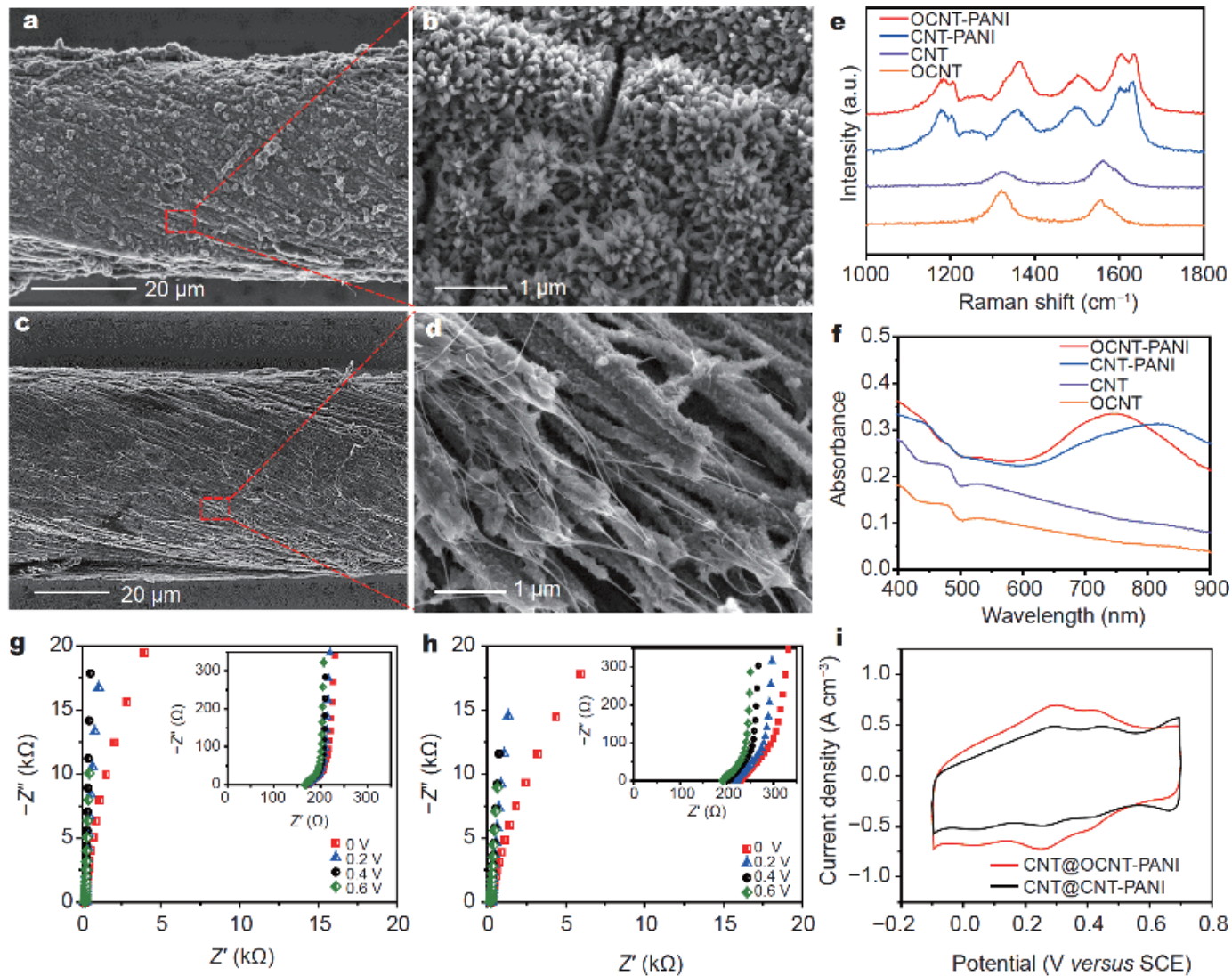

Figure 2 The impact of hydrophilic sheath. SEM images of CNT@CNT-PANI fiber (a, b) and CNT@OCNT-PANI fiber (c, d) with 70 wt.\% PANI at low and high magnifications, respectively. Raman spectra $\left(\lambda_{\text {exc }}=532 \mathrm{~nm}\right)(\mathrm{e})$ and UV-vis spectra (f) of CNTs, OCNTs, CNT-PANI composites and OCNT-PANI composites. Nyquist plots of CNT@CNT-PANI (g) and CNT@OCNT-PANI (h) at different potentials versus SCE. The high-frequency region is highlighted in the inserted panel. (i) CV characterization of CNT@CNT-PANI and CNT@OCNT-PANI electrodes at a scan rate of $10 \mathrm{mV} \mathrm{s}^{-1}$.

$1,320 \mathrm{~cm}^{-1}$ revealed increased defects due to oxygen plasma treatment. OCNT-PANI and CNT-PANI composites, both demonstrated doped PANI in emeraldine salt form including cyclized structure containing nitrogen generated by crosslinking at $1,632 \mathrm{~cm}^{-1}, \mathrm{C}-\mathrm{C}$ stretching of the benzenoid rings at $1,602 \mathrm{~cm}^{-1}, \mathrm{C}=\mathrm{N}$ stretching of the quinoid rings at $1,498 \mathrm{~cm}^{-1}, \mathrm{C}-\mathrm{N}^{+\bullet}$ stretching at $1,361 \mathrm{~cm}^{-1}$, in-plane $\mathrm{C}-\mathrm{H}$ bending of the benzenoid rings at $\sim 1,203 \mathrm{~cm}^{-1}$ and in-plane $\mathrm{C}-\mathrm{H}$ bending of the quinoid rings at $1,177 \mathrm{~cm}^{-1}$. However, as to peaks at $\sim 1,498$ and $\sim 1,177 \mathrm{~cm}^{-1}$ of the quinoid rings, the OCNT-PANI composites showed weakened peaks, suggesting decreased quinoid units. According to previous reports $[21,22]$, the decrease of peak intensity at 1,498 and $1,177 \mathrm{~cm}^{-1}$ of Raman spectra was related to the siteselective interactions between the quinoid units of PANI chain and CNTs, inducing quinoid units chemically transformed to benzenoid units and chain conformation varied from a coil-like polymer to an extended one. The less the peak intensity of quinoid units was, the greater the interactions between PANI and OCNTs were. On the other hand, more oxygen-containing groups on the OCNT surface resulted in more hydrogen bonds between OCNTs and the amino groups of aniline monomers, causing more aniline monomers adsorbed on the surface of OCNTs which acted as the template of PANI [23]. Therefore, the interactions between PANI and OCNT were stronger than those between PANI and CNT. Furthermore, ultraviolet-visible (UV-vis) spectra of CNT, OCNT, CNT-PANI composites and OCNT-PANI composites are compared in Fig. 2f. Both CNTs and OCNTs show no peaks at the wavelength range of $700-900 \mathrm{~nm}$. For the CNT-PANI composites, a strong peak appears at $816 \mathrm{~nm}$, which is assigned to the characteristic absorbance of protonated PANI [24,25]. Accordingly, the characteristic absorbance for OCNT-PANI composites is blue-shifted to $746 \mathrm{~nm}$, indicating enhanced interactions between OCNTs and PANI $[23,26]$. 
Electrochemical characterization was further employed to investigate the CNT@CNT-PANI and CNT@OCNTPANI fibers based on a three-electrode system. Here we chose the fiber electrodes with 30 wt.\% PANI considering that the surface morphology and structure of CNT@CNT-PANI and CNT@OCNT-PANI exhibited obvious differences with the PANI loading over $30 \mathrm{wt} . \%$ (Fig. S4b, e). Nyquist plots at different potentials are presented in Fig. 2g, h. With the increase of potential, the Nyquist plots at low frequency of both samples show steeper slope closer to an ideal capacitive behavior. The higher applied potential is nearer to the redox potentials of PANI shown in the cyclic voltammetry (CV) (Fig. 2i). The CNT@OCNT-PANI demonstrated a more obvious decrease of equivalent series resistance (ESR) (Fig. S6). The PANI was partly oxidized and more quinoid units produced in the PANI backbone as potential increased from 0 to $0.6 \mathrm{~V}$ versus SCE, which facilitated the protonation and finally increased the electrical conductivity of PANI [24]. Due to the improved interactions between OCNTs and PANI, PANI gave priority to protons in the electrolyte and more protonated sites produced in the PANI backbone for CNT@OCNT-PANI. In addition, an extended 45-degree linear region appeared across all potentials in the Nyquist plots of CNT@OCNTPANI compared with CNT@CNT-PANI sample. It is associated with the increased ion diffusion resistance $[27,28]$, which was related to the increased interfacial redox reactions for the CNT@OCNT-PANI electrode (Fig. 2g, h). Furthermore, CNT@OCNT-PANI yielded a higher electrochemical performance than CNT@CNTPANI, with characteristic of an augmented CV integral area (Fig. 2i).

The CNT@CNT-PANI and CNT@OCNT-PANI fibers with different PANI loadings were employed to fabricate fiber-shaped supercapacitors by intertwining two fiber electrodes coated with $\mathrm{PVA} / \mathrm{H}_{3} \mathrm{PO}_{4}$ gel electrolyte. With the increase of PANI from 0 to $70 \mathrm{wt. \%}$ (Figs S7a, S8a), $\mathrm{CV}$ curves demonstrated obvious redox peaks and enlarged integral area. Correspondingly, galvanostatic charge-discharge $(\mathrm{GCD})$ curves showed triangles with prolonged discharge time (Figs S7b, S8b). Specific capacitances were further calculated according to the GCD curves, which were both incremental with the increasing PANI (Fig. S9). Benefiting from the large surface areas of CNTs and OCNTs, the obtained fibers achieved massive accessible PANI for pseudocapacitance utilization, followed by an improved specific capacitance with increasing PANI loading. With the increase of PANI content, the cycle life declined (Fig. S10). After 2,000 cycles, capacitance retention of CNT@OCNT-70\%PANI was about $80 \%$, lower than the $90 \%$ capacitance retention of CNT@OCNT-10\%PANI. For CNT@OCNT-PANI, more exposed PANI were accessible to the ions in the electrolyte during charging and discharging processes, attributing to the hydrophilic surface and the interactions between OCNTs and PANI. Hence, the supercapacitor based on CNT@OCNT-PANI achieved a much greater charge storage capacity with specific capacitance about 1.4 times that of CNT@CNT-PANI counterpart at the 70 wt.\% PANI content (Fig. S11). Typical GCD curves at different current densities and CV curves at different scan rates of the supercapacitor from CNT@OCNT-PANI with 70 wt.\% PANI loading are displayed in Fig. S12.

Although OCNTs could enhance the electrochemical property of the fiber electrode, the increased resistance should not be ignored. As shown in Fig. 3a, CNT@OCNT-PANI fiber shows larger ESR than CNT@CNT-PANI. On the one hand, the structure damage during plasma treatment increases the resistance of resulting fiber. On the other hand, OCNT composites possess more benzenoid units in the PANI backbone as shown in Raman and UV-vis spectroscopy (Fig. 2e, f), which show higher resistance before oxidation at positive potential (Fig. 2g, h). Therefore, Au nanoparticles were incorporated into the core of fiber to improve the electrical conductivity. Compared with bare CNT fibers (Fig. S13a), the Au-modified CNT fibers were larger (Fig. S13c) due to the sufficient sputtering process where $\mathrm{Au}$ nanoparticles were deposited on the CNT sheets uniformly as shown in Fig. S13b, d. Then, OCNT sheets were wound around the as-prepared Au-modified CNT fiber to fabricate CNT-Au@OCNT fiber with hydrophilic OCNTs sheath (Fig. S13e, f). Due to the homogeneous distribution of $\mathrm{Au}$ nanoparticles, electron transport among CNTs was improved and electrical conductivity increased appreciably to $5,800 \mathrm{~S} \mathrm{~cm}^{-1}$ for CNT-Au fiber compared with the only $200 \mathrm{~S} \mathrm{~cm}^{-1}$ for bare CNT fiber (Fig. S14). Hence, CNT-Au@OCNT based supercapacitors could realize a high reversibility with scan rates up to $10,000 \mathrm{mV} \mathrm{s}^{-1}$ (Fig. S15), showing its potential of stable operation at high currents. Nevertheless, without $\mathrm{Au}$ nanoparticles, the CNT@OCNT counterpart could not maintain the rectangle curve at $10,000 \mathrm{mV} \mathrm{s}^{-1}$ (Fig. S16). According to the Nyquist plots of both supercapacitors, incorporation of $\mathrm{Au}$ nanoparticles lowered the ESR tremendously from $383 \Omega$ for CNT@OCNT to $31 \Omega$ for CNT-Au@OCNT (Fig. S17). Similarly, CNT-Au@CNT based supercapacitor demonstrated a much lower ESR of $47 \Omega$ than that of CNT@CNT equivalent $(468 \Omega)$ 

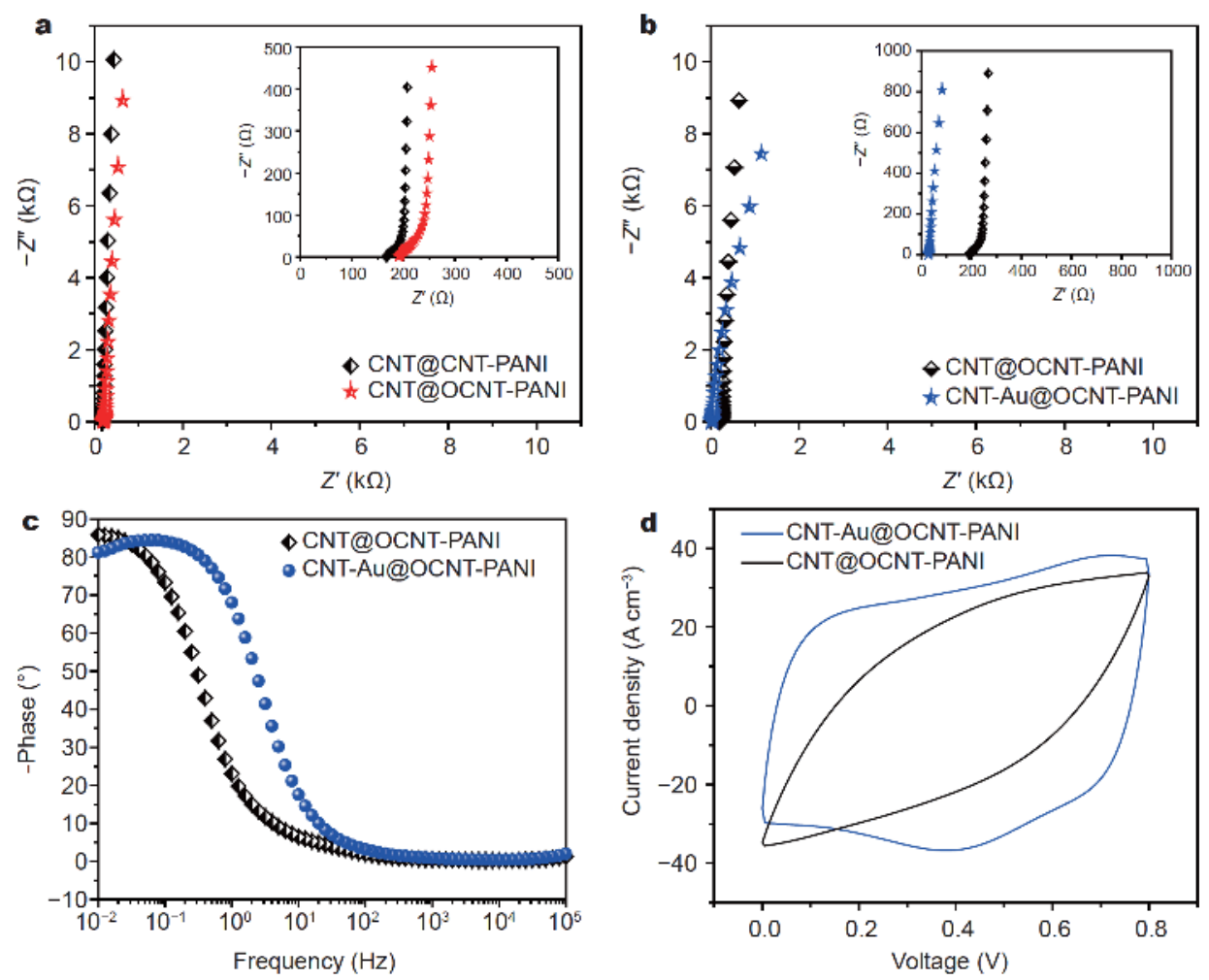

Figure 3 The effect of highly conductive hydrophobic core. Comparison of Nyquist plots of CNT@CNT-PANI and CNT@OCNT-PANI electrodes (a) and CNT@OCNT-PANI and CNT-Au@OCNT-PANI electrodes (b) at 0.6 V versus SCE. The high frequency region is highlighted in the inserted panel. (c) Dependence of impedance phase angle on frequency for CNT@OCNT-PANI and CNT-Au@OCNT-PANI electrodes at 0.6 V versus SCE. (d) CV curves of supercapacitors based on CNT@OCNT-PANI and CNT-Au@OCNT-PANI fibers at a scan rate of $200 \mathrm{mV} \mathrm{s}{ }^{-1}$.

(Fig. S18).

The PANI was deposited on the CNT-Au@OCNT fiber to fabricate CNT-Au@OCNT-PANI electrode. This composite fiber electrode shows smaller ESR than CNT@OCNT-PANI with the same PANI loading (Fig. 3b). Impedance phase angle was further plotted as a function of frequency for CNT@OCNT-PANI and CNT-Au@OCNT-PANI electrodes (Fig. S19). Time constant $\tau_{0}\left(\tau_{0}=1 / f_{0}\right)$ is used to assess the instantaneous energy delivery, which means the minimum time needed to discharge all the energy from the device at an efficiency of over $50 \%[29,30]$. Here, $f_{0}$ denotes the characteristic frequency at the phase angle of $-45^{\circ}$. CNT-Au@OCNTPANI shows a lower $\tau_{0}$ of $0.35 \mathrm{~s}$ than that of CNT@OCNT-PANI (2.78 s), indicating improved energy delivery at larger current (Fig. 3c). As a result, the supercapacitor based on CNT-Au@OCNT-PANI demonstrates a higher capacitance with improved reversibility than the counterpart at a large scan rate of $200 \mathrm{mV} \mathrm{s}^{-1}$ (Fig. 3d). Similar conclusion could be drawn for CNTAu@CNT-PANI compared with CNT@CNT-PANI
(Figs S20 and S21).

Benefiting from the core-sheath structure of the composite electrode and the synergistic effect among each component, the resulting supercapacitor shows excellent reversible pseudocapacitive behavior from 10 to $1,000 \mathrm{mV} \mathrm{s}^{-1}$ (Fig. 4a). The current densities of supercapacitors based on CNT-Au@OCNT-PANI, CNT@OCNT-PANI and CNT-Au@CNT-PANI are shown in Fig. 4b with the scan rate from 10 to $5,000 \mathrm{mV} \mathrm{s}^{-1}$. The current densities of CNTAu@OCNT-PANI show a linear relationship from 10 to $1,000 \mathrm{mV} \mathrm{s}^{-1}$ with a slow departure from the linear region as the scan rates exceed $1,000 \mathrm{mV} \mathrm{s}^{-1}$, deriving from the limited ion diffusion at higher scan rates. However, the CNT@OCNT-PANI and CNT-Au@CNT-PANI based supercapacitors show a narrower linear region from 10 to $200 \mathrm{mV} \mathrm{s}^{-1}$ followed by a drastic deviation. And the CNT-Au@OCNT-PANI based supercapacitor shows a much smaller ESR of $36 \Omega$ than CNT@OCNT-PANI $(255 \Omega)$ and CNT-Au@CNT-PANI (109 $\Omega$ ) (Fig. 4c). Moreover, the specific capacitances were compared and 

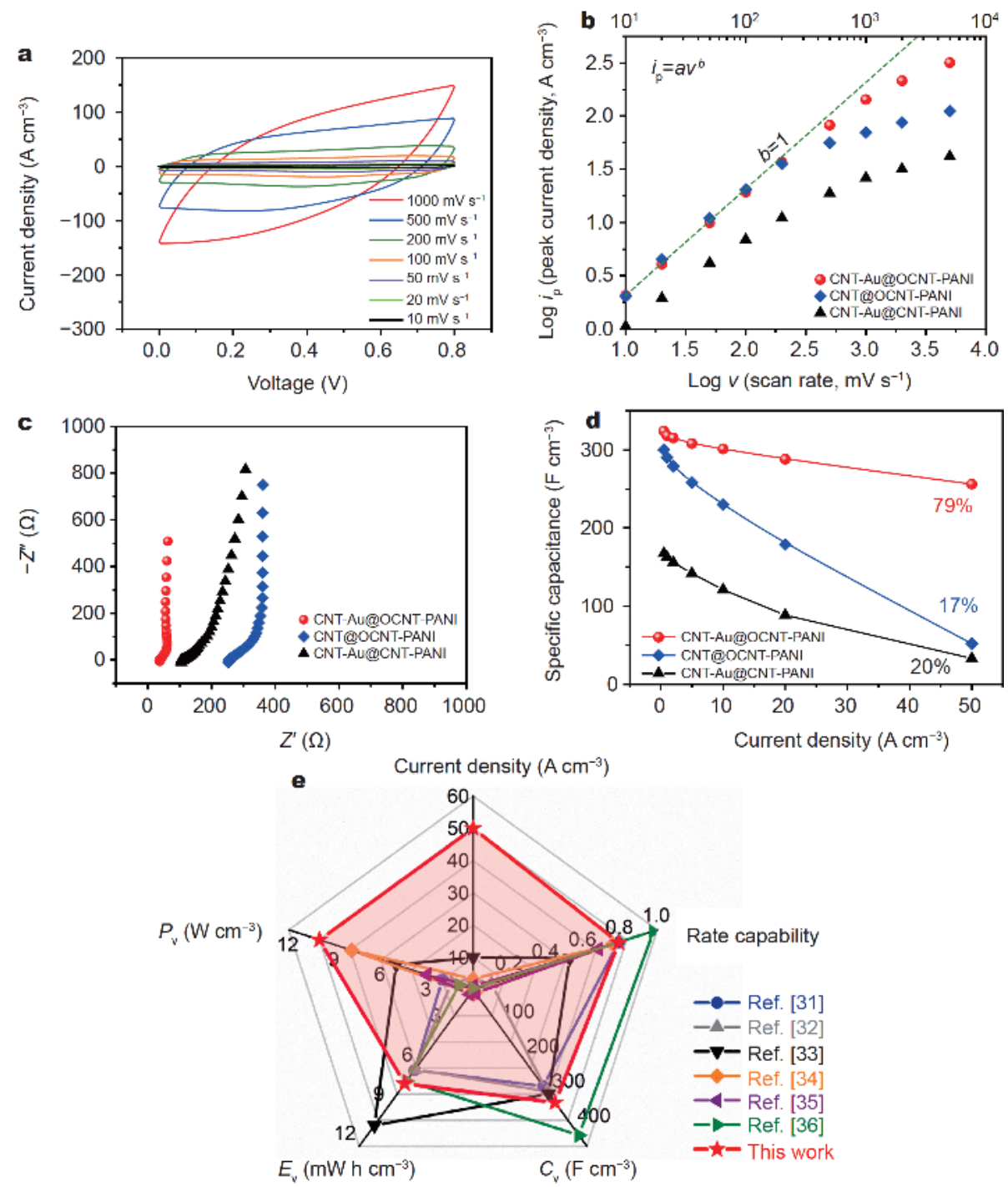

Figure 4 Fiber-shaped supercapacitors based on the amphiphilic core-sheath structured composite fibers using gel electrolyte. (a) CV measurement of the supercapacitor using CNT-Au@OCNT-PANI electrodes from 10 to $1,000 \mathrm{mV} \mathrm{s}^{-1}$. Comparison of the dependence of current density derived from CV curves on scan rate from 10 to $5,000 \mathrm{mV} \mathrm{s}^{-1}$ (b) and Nyquist plots (c) of fiber-shaped supercapacitors based on CNT-Au@OCNT-PANI, CNT@OCNT-PANI and CNT-Au@CNT-PANI. (d) Specific capacitance as a function of current density from 0.5 to 50 A cm ${ }^{-3}$ for CNT-Au@OCNTPANI, CNT@OCNT-PANI and CNT-Au@CNT-PANI. (e) Comparison of this work with other reported fiber-shaped supercapacitors based on gel electrolyte. The rate capability referred to the capacitance ratio at the maximal current density versus minimal current density.

plotted as a function of current density ranged from 0.50 to $50 \mathrm{~A} \mathrm{~cm}^{-3}$ (Fig. 4d). And the maximal specific capacitance of CNT-Au@OCNT-PANI was calculated as $324 \mathrm{~F} \mathrm{~cm}^{-3}\left(118 \mathrm{~F} \mathrm{~g}^{-1}\right)$ at $0.50 \mathrm{~A} \mathrm{~cm}^{-3}$ which was higher than that of CNT@OCNT-PANI $\left(299 \mathrm{~F} \mathrm{~cm}^{-3}\right)$ and much higher than that of CNT-Au@CNT-PANI $\left(168 \mathrm{~F} \mathrm{~cm}^{-3}\right)$. The obviously lower capacitance of CNT-Au@CNTPANI could be attributed to the aggregated PANI on the hydrophobic CNT sheath less accessible to electrolyte ions, which also corresponds to the lower current densities in the CV curves (Fig. 4b). Notably, CNTAu@OCNT-PANI demonstrates high rate capability with a capacitance retention of $79 \%\left(256 \mathrm{~F} \mathrm{~cm}^{-3}\right)$ at $50 \mathrm{~A} \mathrm{~cm}^{-3}$. By contrast, CNT@OCNT-PANI could only maintain $17 \%$ of its maximal capacitance $\left(51 \mathrm{~F} \mathrm{~cm}^{-3}\right)$. CNTAu@CNT-PANI also shows a low capacitance retention of $20 \%\left(34 \mathrm{~F} \mathrm{~cm}^{-3}\right)$ in spite of $\mathrm{Au}$ incorporation because rate capability is associated with both electron transport and ion accessibility. Moreover, the fiber-shaped supercapacitor from CNT-Au@OCNT-PANI demonstrates 

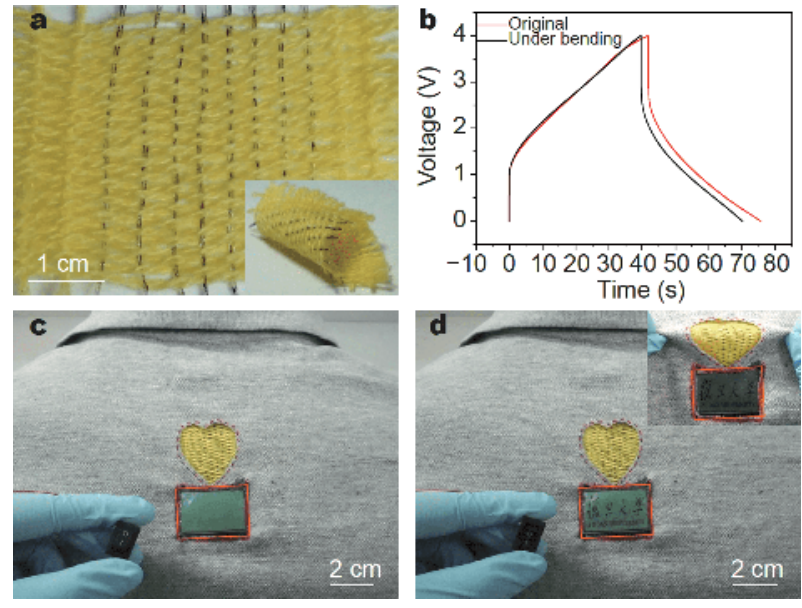

Figure 5 Application demonstration of energy textile with the fibershaped supercapacitors. (a) An energy textile woven from fiber-shaped supercapacitors (a length of $4 \mathrm{~cm}$ and diameter of $1 \mathrm{~mm}$ on average per supercapacitor) and cotton threads. The inset shows the flexibility of the resulting energy textile. (b) GCD curves of an energy textile in original flat state and under bending. The energy textile comprised four sets of fiber-shaped supercapacitors in series with each set including two fibershaped supercapacitors in parallel. (c) The textile in (a) integrated into a piece of common clothes and then connected to an LCD. (d) Once the circuit closed, the LCD showing the name of Fudan University powered by the energy textile. Note that the LCD can work stably even when the energy textile is buckled as shown in inset.

high energy density and power density of $7.2 \mathrm{~mW} \mathrm{~h} \mathrm{~cm}^{-3}$ $\left(2.6 \mathrm{~mW} \mathrm{~h} \mathrm{~g}^{-1}\right)$ and $10 \mathrm{~W} \mathrm{~cm}^{-3}\left(3.6 \mathrm{~W} \mathrm{~g}^{-1}\right)$, respectively. Compared with other fiber-shaped supercapacitors based on gel electrolyte [31-36], the as-prepared supercapacitor presents the best comprehensively volumetric performances (Fig. 4e). Furthermore, Fig. S22 illustrates the Ragone plots of the fiber-shaped supercapacitor, compared with the corresponding values of previous reports. The moderate gravimetric power and energy densities are due to the huge deposition of Au nanoparticles whose mass accounts for over $60 \%$ of the electrodes. Considering the superb energy density of asymmetrical supercapacitor, i.e., a flexible non-woven fabrics supercapacitor delivering high energy density of $96.5 \mathrm{~mW} \mathrm{~h} \mathrm{~cm}^{-3}$ via a microfluidic-spinning technique [37], the as-prepared composite fiber can be thus designed for asymmetrical supercapacitor to further enhance the electrochemical performances. In addition, the supercapacitor could maintain $85 \%$ capacitance even after 10,000 cycles of charging and discharging (Fig. S23).

Furthermore, we investigated the flexibility and applications of the fiber-shaped supercapacitors. Firstly, the mechanical properties of the as-prepared fiber electrodes were tested (Fig. S24). The incorporation of
OCNTs which had more defects than CNTs decreased the tensile strength. So did the incorporation of $\mathrm{Au}$ nanoparticles in the CNT core, which was due to the expanded gaps and weakened interactions among CNT bundles caused by $\mathrm{Au}$ nanoparticles. Besides, the increased diameter of the composite fiber also resulted in the degradation of tensile strength (Fig. S13). Hence, the resulting fiber-shaped supercapacitor based on CNTAu@OCNT-PANI shows mediocre flexibility. After bending at $90^{\circ}$ for 3,000 cycles, $15 \%$ of capacitance loss generated (Fig. S25). As a demonstration for wearable electronic applications, four groups of supercapacitors connected in series were woven with cotton threads to fabricate an energy textile with each group containing two supercapacitors in parallel (Fig. 5a). The energy textile could be bent into different shapes such as a cylinder (inset in Fig. 5a), suggesting its good flexibility. Likewise, electrochemical characterization of the energy textile under bending demonstrated a little degradation compared with original flat state at the same current (Fig. 5b). After integration into a piece of common clothes, the heart-shaped energy textile was connected to a liquid crystal displayer (LCD), using a switch to control the on-off of the energy textile (Fig. $5 \mathrm{c}$ ). When the switch was on, the LCD was powered by the energy textile and remained steadily under buckling of the energy textile (Fig. 5d).

\section{CONCLUSION}

In summary, a new family of amphiphilic core-sheath structured CNT-Au@OCNT-PANI fiber electrodes has been developed. The electron transport and ion accessibility of the composite fiber have been simultaneously enhanced by the core-sheath structure and interface optimization. A high specific capacitance of $324 \mathrm{~F} \mathrm{~cm}^{-3}$ was achieved at $0.50 \mathrm{~A} \mathrm{~cm}^{-3}$, which could be maintained by $79 \%$ at a high rate of $50 \mathrm{~A} \mathrm{~cm}^{-3}$. Moreover, the fiber-shaped supercapacitor derived from CNTAu@OCNT-PANI demonstrated a high energy density and power density of $7.2 \mathrm{~mW} \mathrm{~h} \mathrm{~cm}^{-3}$ and $10 \mathrm{~W} \mathrm{~cm}^{-3}$, respectively. The strategy of structure design and material utilization can be promoted to other energy storage systems including but not limited to lithium-ion and metal-air batteries.

\section{Received 25 December 2018; accepted 15 February 2019;} published online 15 March 2019

1 Wu G, Tan P, Wu X, et al. High-performance wearable microsupercapacitors based on microfluidic-directed nitrogen-doped graphene fiber electrodes. Adv Funct Mater, 2017, 27: 1702493 
2 Wang L, Wang L, Zhang Y, et al. Weaving sensing fibers into electrochemical fabric for real-time health monitoring. Adv Funct Mater, 2018, 28: 1804456

3 Liu P, Gao Z, Xu L, et al. Polymer solar cell textiles with interlaced cathode and anode fibers. J Mater Chem A, 2018, 6: 19947-19953

4 Jia R, Li L, Ai Y, et al. Self-healable wire-shaped supercapacitors with two twisted $\mathrm{NiCo}_{2} \mathrm{O}_{4}$ coated polyvinyl alcohol hydrogel fibers. Sci China Mater, 2018, 61: 254-262

5 Lee JA, Shin MK, Kim SH, et al. Ultrafast charge and discharge biscrolled yarn supercapacitors for textiles and microdevices. Nat Commun, 2013, 4: 1970

6 Sun H, Zhang Y, Zhang J, et al. Energy harvesting and storage in 1D devices. Nat Rev Mater, 2017, 2: 17023

7 Kou L, Huang T, Zheng B, et al. Coaxial wet-spun yarn supercapacitors for high-energy density and safe wearable electronics. Nat Commun, 2014, 5: 3754

8 Liang Y, Wang Z, Huang J, et al. Series of in-fiber graphene supercapacitors for flexible wearable devices. J Mater Chem A, 2015, 3: $2547-2551$

9 Lima MD, Fang S, Lepró X, et al. Biscrolling nanotube sheets and functional guests into yarns. Science, 2011, 331: 51-55

10 Chen $\mathrm{P}, \mathrm{Xu} \mathrm{Y}, \mathrm{He} \mathrm{S}$, et al. Hierarchically arranged helical fibre actuators driven by solvents and vapours. Nat Nanotech, 2015, 10: 1077-1083

11 Ren J, Zhang Y, Bai W, et al. Elastic and wearable wire-shaped lithium-ion battery with high electrochemical performance. Angew Chem Int Ed, 2014, 53: 7864-7869

12 Sun $\mathrm{H}, \mathrm{Fu} \mathrm{X}$, Xie S, et al. Electrochemical capacitors with high output voltages that mimic electric eels. Adv Mater, 2016, 28: 2070-2076

13 Wang X, Zhang Q, Sun J, et al. Facile synthesis of hierarchical porous manganese nickel cobalt sulfide nanotube arrays with enhanced electrochemical performance for ultrahigh energy density fiber-shaped asymmetric supercapacitors. J Mater Chem A, 2018, 6: $8030-8038$

14 Zhou J, Chen N, Ge Y, et al. Flexible all-solid-state micro-supercapacitor based on $\mathrm{Ni}$ fiber electrode coated with $\mathrm{MnO}_{2}$ and reduced graphene oxide via electrochemical deposition. Sci China Mater, 2018, 61: 243-253

$15 \mathrm{Wu} \mathrm{X}, \mathrm{Wu}$ G, Tan P, et al. Construction of microfluidic-oriented polyaniline nanorod arrays/graphene composite fibers for application in wearable micro-supercapacitors. J Mater Chem A, 2018, 6: $8940-8946$

16 Qin T, Peng S, Hao J, et al. Flexible and wearable all-solid-state supercapacitors with ultrahigh energy density based on a carbon fiber fabric electrode. Adv Energy Mater, 2017, 7: 1700409

17 Meng J, Nie W, Zhang K, et al. Enhancing electrochemical performance of graphene fiber-based supercapacitors by plasma treatment. ACS Appl Mater Interfaces, 2018, 10: 13652-13659

18 Gao L, Song J, Surjadi JU, et al. Graphene-bridged multifunctional flexible fiber supercapacitor with high energy density. ACS Appl Mater Interfaces, 2018, 10: 28597-28607

19 Shi P, Chen R, Hua L, et al. Highly concentrated, ultrathin nickel hydroxide nanosheet ink for wearable energy storage devices. Adv Mater, 2017, 29: 1703455

$20 \mathrm{Fu} \mathrm{X}$, Sun $\mathrm{H}$, Xie S, et al. A fiber-shaped solar cell showing a record power conversion efficiency of 10\%. J Mater Chem A, 2018, 6: 4551

21 Cochet M, Maser WK, Benito AM, et al. Synthesis of a new polyaniline/nanotube composite: "in-situ" polymerisation and charge transfer through site-selective interaction. Chem Commun, 2001, 0: 1450-1451

22 Yao Q, Chen L, Zhang W, et al. Enhanced thermoelectric performance of single-walled carbon nanotubes/polyaniline hybrid nanocomposites. ACS Nano, 2010, 4: 2445-2451

$23 \mathrm{Wu}$ TM, Lin YW, Liao CS. Preparation and characterization of polyaniline/multi-walled carbon nanotube composites. Carbon, 2005, 43: 734-740

24 Wan M. Absorption spectra of thin film of polyaniline. J Polym Sci A Polym Chem, 1992, 30: 543-549

25 Chen X, Lin H, Chen P, et al. Smart, stretchable supercapacitors. Adv Mater, 2014, 26: 4444-4449

26 Liu C, Chen F, Zhang J, et al. Raman spectroscopic studies on electrosynthesized polyaniline film. Acta Phys-Chim Sin, 2003, 19: 810-814

27 Lukatskaya MR, Kota S, Lin Z, et al. Ultra-high-rate pseudocapacitive energy storage in two-dimensional transition metal carbides. Nat Energy, 2017, 2: 17105

28 Wang $\mathrm{Y}$, Guo CX, Liu J, et al. $\mathrm{CeO}_{2}$ nanoparticles/graphene nanocomposite-based high performance supercapacitor. Dalton Trans, 2011, 40: 6388-6391

29 Wu ZS, Parvez K, Feng X, et al. Graphene-based in-plane microsupercapacitors with high power and energy densities. Nat Commun, 2013, 4: 2487

30 Taberna PL, Simon P, Fauvarque JF. Electrochemical characteristics and impedance spectroscopy studies of carbon-carbon supercapacitors. J Electrochem Soc, 2003, 150: A292

31 Tao J, Liu N, Ma W, et al. Solid-state high performance flexible supercapacitors based on polypyrrole- $\mathrm{MnO}_{2}$-carbon fiber hybrid structure. Sci Rep, 2013, 3: 2286

32 Liu L, Yu Y, Yan C, et al. Wearable energy-dense and power-dense supercapacitor yarns enabled by scalable graphene-metallic textile composite electrodes. Nat Commun, 2015, 6: 7260

33 Li GX, Hou PX, Luan J, et al. A $\mathrm{MnO}_{2}$ nanosheet/single-wall carbon nanotube hybrid fiber for wearable solid-state supercapacitors. Carbon, 2018, 140: 634-643

34 Sun Y, Sills RB, Hu X, et al. A bamboo-inspired nanostructure design for flexible, foldable, and twistable energy storage devices. Nano Lett, 2015, 15: 3899-3906

35 Yu D, Zhai S, Jiang W, et al. Transforming pristine carbon fiber tows into high performance solid-state fiber supercapacitors. Adv Mater, 2015, 27: 4895-4901

36 Padmajan Sasikala S, Lee KE, Lim J, et al. Interface-confined high crystalline growth of semiconducting polymers at graphene fibers for high-performance wearable supercapacitors. ACS Nano, 2017, 11: 9424-9434

$37 \mathrm{Wu} \mathrm{X}, \mathrm{Xu} \mathrm{Y}, \mathrm{Hu} \mathrm{Y}$, et al. Microfluidic-spinning construction of black-phosphorus-hybrid microfibres for non-woven fabrics toward a high energy density flexible supercapacitor. Nat Commun, 2018, 9: 4573

Acknowledgements This work was supported by the Ministry of Science and Technology (2016YFA0203302), the National Natural Science Foundation of China (21634003, 51573027, 51673043, 21604012, 21805044 and 21875042), Shanghai Science and Technology Committee (16JC1400702, 17QA1400400, 18QA1400700 and 18QA1400800), SHMEC (2017-01-07-00-07-E00062) and Yanchang Petroleum Group. Part of the sample fabrication was performed at Fudan Nano-Fabrication Laboratory. 
Author contributions $\mathrm{Fu} \mathrm{X}$, Wang $\mathrm{B}$ and Peng $\mathrm{H}$ designed the experiments. $\mathrm{Fu} \mathrm{X}, \mathrm{Li} \mathrm{Z}$ and $\mathrm{Xu} \mathrm{L}$ performed the experiments with supports and suggestions from Liao M, Xie S, Sun H and Sun X. All authors contributed to the general discussion.
Conflict of interest The authors declare no conflict of interest.

Supplementary information Supporting data is available in the online version of the paper.

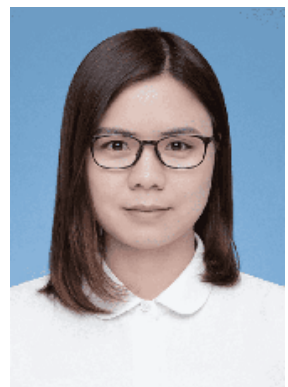

Xuemei Fu received her BE degree in polymer materials and engineering from Nanchang University in 2014. She is currently a $\mathrm{PhD}$ candidate majored in macromolecular chemistry and physics under the supervision of Prof. Huisheng Peng at Fudan University. Her research is mainly focused on the synthesis of carbon nanomaterials and their applications in flexible energy conversion and storage devices.
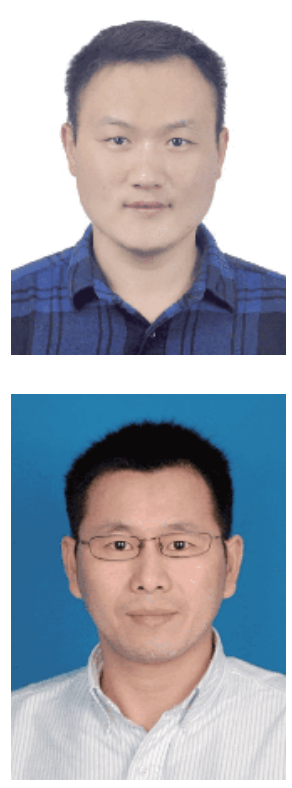

Bingjie Wang received his BE degree in polymer materials and engineering at Sichuan University in 2009, and his PhD in polymer chemistry and physics at Ningbo Institute of Materials Technology and Engineering, Chinese Academy of Sciences in 2014. He is currently an associate professor in the Laboratory of Advanced Materials at Fudan University, China. His research focuses on the smart fibers for energy and electronics.
Huisheng Peng received his BE degree in polymer materials at Donghua University in 1999, his MSc in macromolecular chemistry and physics at Fudan University in 2003, and his PhD in chemical engineering at Tulane University in 2006. He then worked at Los Alamos National Laboratory from 2006 to 2008. He is currently a full professor in the Department of Macromolecular Science and Laboratory of Advanced Materials at Fudan University, China. His research focuses on the smart fibers for energy and electronics.

\title{
具有优异综合性能的双亲性核壳结构复合纤维超级电容器
}

\author{
付雪梅 ${ }^{1}$, 黎卓尔 ${ }^{1}$, 许黎敏 $^{1}$, 廖萌 $^{1}$, 孙浩 $^{2}$, 解松林 $^{1}$, 孙雪梅 $^{1}$, 王兵杰 ${ }^{1 *}$, 彭慧胜 ${ }^{*}$
}

摘要 纤维状超级电容器是柔性储能器件的一个重要分支, 被学术界和产业界广泛关注. 如何制备高性能纤维电极及器件是目前的研究 重点之一. 针对这一问题, 我们制备了一类具有双亲性核壳结构的碳纳米管复合纤维电极. 该复合纤维电极的壳层为聚苯胺修饰的亲水碳 纳米管以实现更好的离子可接近性, 从而有效提升电极的电化学性能; 而核层为纳米金沉积疏水碳纳米管以实现快速电子传输, 从而显著 提高电极的电导率. 得益于各组分之间的协同效应, 在 $0.5 \mathrm{~A} \mathrm{~cm}^{-3}$ 的电流密度下, 该复合纤维电极的比容量可以达到 $324 \mathrm{~F} \mathrm{~cm}^{-3}$. 同时该纤 维电极也展示了优异的倍率性能, 在 $50 \mathrm{~A} \mathrm{~cm}^{-3}$ 电流密度下, 比容量可以保持为小电流下比容量的 $79 \%$ (即 $256 \mathrm{~F} \mathrm{~cm}^{-3}$ ). 由此得到的纤维状 超级电容器也实现了高能量密度和高功率密度, 分别可达到 $7.2 \mathrm{~mW} \mathrm{~h} \mathrm{~cm}$ 和 $10 \mathrm{~W} \mathrm{~cm}^{-3}$. 这种多层次的复合电极设计为制备其他高性能 可穿戴器件提供了一种可行的方法. 